\title{
INEQUALITY OF TWO CRITICAL PROBABILITIES FOR PERCOLATION
}

\author{
JEFF KAHN ${ }^{1}$
}

Department of Mathematics, Rutgers University, Piscataway NJ 08854

email: jkahn@math.rutgers.edu

Submitted 5 September 2003, accepted in final form 5 September 2003

AMS 2000 Subject classification: 60CO5, 82B43

Keywords: percolation, critical probability

Abstract

For a locally finite, connected graph $G$ with distinguished vertex 0 , let $p_{c}(G)$ be the usual critical probability for bond percolation on $G$, and

$$
p_{\text {cut }}(G)=\sup \left\{p: \inf _{\Pi} \mathrm{E}_{p}|C(0) \cap \Pi|=0\right\}\left(\leq p_{c}\right),
$$

where $\Pi$ ranges over cutsets (sets of vertices "separating 0 from $\infty$ "), $\mathrm{E}_{p}$ refers to (Bernoulli bond) percolation with $p$ the probability that an edge is open, and $C(0)$ is the open cluster containing 0 .(The definition is easily seen to be independent of the choice of distinguished vertex.)

We disprove a conjecture of Russ Lyons stating that $p_{\text {cut }}(G)=p_{c}(G)$ for every $G$, and propose a possible alternative.

\section{Introduction}

We consider the usual (Bernoulli) bond percolation on a locally finite, connected graph $G=$ $(V, E)$ : each $e \in E$ is "open" with probability $p$, these choices made independently. We write $H$ for the resulting random subgraph of $G$ and $\operatorname{Pr}_{p}$ for the law of $H$. (For percolation background, see e.g. [2] or [5].)

For $X, Y \subseteq V$ we denote by $\{X \longleftrightarrow Y\}$ the event that some $x \in X$ and $y \in Y$ are in the same component of $H$ (of course $\{x \longleftrightarrow y\}$ means $\{\{x\} \longleftrightarrow\{y\}\}$ ), and by $\{x \longleftrightarrow \infty\}$ the event that the component of $x$ in $H$ - denoted $C(x)$ - is infinite.

The standard critical probability for bond percolation is

$$
p_{c}=p_{c}(G)=\sup \left\{p: \operatorname{Pr}_{p}(H \text { contains an infinite component })=0\right\} .
$$

(For connected $G$ this is easily seen to coincide with $\sup \left\{p: \operatorname{Pr}_{p}(|C(x)|=\infty)=0\right\}$ for any $x \in V$.) Our focus here is on a second critical probability, introduced by Russ Lyons in [3].

\footnotetext{
${ }^{1}$ SUPPORTED IN PART BY NSF GRANT DMS-0200856.
} 
Given $0 \in V$, say $\Pi \subseteq V$ is a cutset for 0 if the component of 0 in $G-\Pi$ is finite (" $\Pi$ separates 0 from infinity"), and set

$$
p_{\text {cut }}(G)=\sup \left\{p: \inf _{\Pi} \mathrm{E}_{p}|C(0) \cap \Pi|=0\right\} .
$$

Again this is easily seen not to depend on our choice of the distinguished vertex. Here we will keep 0 fixed throughout, and $\Pi$ will always range over cutsets for 0 .

Note $p_{\text {cut }} \leq p_{c}$ is trivial because of the "first moment method" bound

$$
\operatorname{Pr}(0 \longleftrightarrow \infty)=\inf _{\Pi} \operatorname{Pr}(0 \longleftrightarrow \Pi) \leq \inf _{\Pi} \mathrm{E}|C(0) \cap \Pi|
$$

Lyons proved $p_{\text {cut }}=p_{c}$ for trees [3] and for certain "tree-like" graphs [4], and suggested ([3], [4]) the possibility that $p_{\text {cut }}$ and $p_{c}$ coincide for every $G$. That equality also holds for the Euclidean cubic lattices follows from [1], [6].

Here we give a simple family of graphs for which equality does not hold, and, in Section 3, suggest a possible salvage.

\section{Example}

A graph $G$ is said to be spherically symmetric about $0 \in V$ if $(\operatorname{Aut}(G))_{0}$ is transitive on

$$
\Pi_{n}:=\{v: d(0, v)=n\}
$$

for each $n$. For such $G$, dealing with $p_{\text {cut }}$ is simplified by the following observation.

Lemma 2.1 If $G$ is spherically symmetric then (for any $p$ )

$$
\inf _{\Pi} \mathrm{E}_{p}|C(0) \cap \Pi|=\inf _{n} \mathrm{E}_{p}\left|C(0) \cap \Pi_{n}\right| .
$$

Proof. Fix a cutset $\Pi$, say with $\Pi \subseteq \Pi_{0} \cup \cdots \cup \Pi_{m}$ (of course it is enough to consider minimal $\Pi$ 's, which are necessarily finite).

Let $P$ be chosen uniformly from the set of $\left(0, \Pi_{m}\right)$-paths of length $m$. By spherical symmetry we have $\operatorname{Pr}(v \in P)=\left|\Pi_{k}\right|^{-1}$ for each $k \leq m$ and $v \in \Pi_{k}$.

So setting $\alpha_{k}=\left|\Pi \cap \Pi_{k}\right| /\left|\Pi_{k}\right|$, we have

$$
1=\operatorname{Pr}(P \cap \Pi \neq \emptyset) \leq \sum_{k \leq m} \mathrm{E}\left|P \cap \Pi \cap \Pi_{k}\right|=\sum_{k \leq m} \alpha_{k},
$$

and

$$
\mathrm{E}|C(0) \cap \Pi|=\sum_{k} \alpha_{k} \mathrm{E}\left|C(0) \cap \Pi_{k}\right| \geq \inf _{n} \mathrm{E}\left|C(0) \cap \Pi_{n}\right|
$$

\section{Construction}

Set $n_{0}=1$ and let $n_{1}, n_{2}, \ldots$ be integers with $n_{i} \geq 5 n_{i-1}$ for $i \geq 1$. Set $m_{i}=n_{1}+\cdots+n_{i}$. The $m_{i}$ th levels play a special role, and we abbreviate $\Pi_{m_{i}}=\Sigma_{i}$. The structure of $G$ is as follows.

For each $i, \Sigma_{i}$ is a clique of size $2^{n_{i}}$, and $G\left[\cup\left\{\Pi_{n}: m_{i-1} \leq n \leq m_{i}\right\}\right]$ consists of the cliques at levels $m_{i-1}$ and $m_{i}$ together with, for each $v \in \Sigma_{i-1}, 2^{n_{i}-n_{i-1}}$ paths of length $n_{i}$ joining $v$ to $\Sigma_{i}$, the resulting $2^{n_{i}}$ paths being disjoint except where they share an end in $\Sigma_{i-1}$. 


\section{Analysis}

It is easy to see that $p_{c}(G) \geq 1 / 2$ : with $B_{i}=\left\{\Sigma_{i-1} \longleftrightarrow \Sigma_{i}\right\}$ we have

$$
\operatorname{Pr}_{p}(0 \longleftrightarrow \infty)<\operatorname{Pr}_{p}\left(B_{i}\right)<\left|\Sigma_{i}\right| p^{n_{i}}=(2 p)^{n_{i}},
$$

which for $p<1 / 2$ tends to zero as $i \rightarrow \infty$.

(Actually $p_{c}(G)=\exp _{2}\left[-\liminf n_{i} / m_{i}\right] \quad\left(=1 / 2\right.$ if $\left.n_{i} / m_{i} \rightarrow 1\right)$ : as shown in [3], for spherically symmetric trees $T$ one has

$$
p_{c}(T)=\left[\liminf _{n}\left|\Pi_{n}\right|^{1 / n}\right]^{-1}
$$

(the expression in brackets is the growth number of $T$ ); and in our case this gives $\left(p_{c}(G) \leq\right.$ ) $p_{c}(T)=\exp _{2}\left[-\liminf n_{i} / m_{i}\right]$ for $T=\left(V(G), E^{\prime}\right)$, where $E^{\prime}$ consists of those edge of $G$ not lying in any $\Sigma_{i}$.)

We assert, on the other hand, that

$$
p_{\text {cut }}(G) \leq .4 \text {. }
$$

(The bound is by no means best possible, but there seems no point in trying to optimize either the construction or the analysis, and we will always opt for crude but convenient estimates in what follows.)

According to Lemma 2.1, (2) can be established by showing that for

$$
p=(1-\varepsilon) / 2 \quad \text { with } \quad \varepsilon \leq 1 / 5
$$

and any $n$,

$$
\mathrm{E}_{p}\left|C(0) \cap \Pi_{n}\right|>1 \text {. }
$$

Proof. Fix $\varepsilon, p$ as in (3), and write $\operatorname{Pr}$ and $\mathrm{E}$ for $\operatorname{Pr}_{p}$ and $\mathrm{E}_{p}$. For $B_{i}$ as above,

$$
\operatorname{Pr}\left(B_{i}\right)>\left|\Sigma_{i}\right| p^{n_{i}}-\left(\begin{array}{c}
\left|\Sigma_{i}\right| \\
2
\end{array}\right) p^{2 n_{i}}>\frac{1}{2}(1-\varepsilon)^{n_{i}} .
$$

Let $C_{i}=\left\{H\left[\Sigma_{i}\right]\right.$ is connected $\}$ and $\xi_{i}=1-\operatorname{Pr}\left(C_{i}\right)$. A routine calculation gives (say)

$$
\xi_{i}<(1+o(1))\left|\Sigma_{i}\right|((1+\varepsilon) / 2)^{\left|\Sigma_{i}\right|-1}<(3 / 4)^{2^{n_{i}}} .
$$

Set $D_{i}=\left\{0 \longleftrightarrow x \quad \forall x \in \Sigma_{i}\right\}$. Since $D_{i} \supseteq \cap_{j=1}^{i}\left(B_{j} \cap C_{j}\right)$ and the $B_{j}$ 's and $C_{j}$ 's are independent, (5) gives

$$
\operatorname{Pr}\left(D_{i}\right)>2^{-i}(1-\varepsilon)^{m_{i}} \prod_{j=1}^{i}\left(1-\xi_{j}\right) .
$$

Now let $n=m_{i}-k$ with $0 \leq k<n_{i}$. For $v \in \Pi_{n}$,

$$
\operatorname{Pr}(0 \longleftrightarrow v)>\operatorname{Pr}\left(D_{i-1}\right) \max \left\{p^{n_{i}-k}, \operatorname{Pr}\left(B_{i}\right)\left(1-\xi_{i}\right) p^{k}\right\}=: \beta_{n} .
$$

The first expression in brackets is of course the probability that the "direct" (i.e. unique shortest) path from $\Sigma_{i-1}$ to $v$ is open. The second is a lower bound on $\operatorname{Pr}\left(B_{i} \cap C_{i} \cap F_{v}\right)$, where $F_{v}$ is the event that the direct path from $\Sigma_{i}$ to $v$ is open (note $\operatorname{Pr}\left(F_{v} \mid B_{i} \cap C_{i}\right) \geq \operatorname{Pr}\left(F_{v}\right)$ ). Then (for instance) $\beta_{n}>(1 / 2)(1-\varepsilon)^{4 n_{i} / 3} 2^{-n_{i} / 2}$ (since $\operatorname{Pr}\left(D_{i-1}\right)>(1-\varepsilon)^{n_{i} / 3}$ and the maximum in $(7)$ is at least $\left.\left(p^{n_{i}} \operatorname{Pr}\left(B_{i}\right)\left(1-\xi_{i}\right)\right)^{1 / 2}>(1 / 2)(1-\varepsilon)^{n_{i}} 2^{-n_{i} / 2}\right)$, yielding, finally,

$$
\mathrm{E}\left|C(0) \cap \Pi_{n}\right|>\left|\Pi_{n}\right| \beta_{n}=2^{n_{i}} \beta_{n}>(1 / 2)(1-\varepsilon)^{4 n_{i} / 3} 2^{n_{i} / 2}>1 .
$$


Remark. As Russ Lyons points out, our construction can easily be modified to have bounded degree; roughly: replace the cliques by expanders and, for each $v \in \Sigma_{i-1}$, replace the paths from $v$ to $\Sigma_{i}$ by a tree of the same depth and with the same leaves, having large (but constant) degrees near the root and paths following this initial stage. We omit the details.

\section{A possible substitute}

Following [5], we regard equality of $p_{\text {cut }}(G)$ and $p_{c}(G)$ as saying that the first moment bound (1) correctly identifies the critical probability for bond percolation on $G$. The construction of Section 2 violates this in the natural way, by arranging that the events $\{0 \longleftrightarrow v\}, v$ in some cutset $\Pi$, are heavily dependent, so that $|C(0) \cap \Pi|=\sum_{v \in \Pi} \mathbf{1}_{\{0 \longleftrightarrow v\}}$, while usually zero, has a large expectation. This is achieved by making connections within $\Pi$ easy, so that once we do reach at least one $v \in \Pi$ (an unlikely event), we then typically reach the rest of $\Pi$ via $v$. So a natural way to try to salvage the conjecture is to disallow the effects of such "internal" connections, as follows.

For $\Pi$ a cutset and $v \in \Pi$, let $A(v, \Pi)$ be the event that there is an open $(0, v)$-path disjoint from $\Pi \backslash\{v\}$. Then we again have a first moment bound,

$$
\operatorname{Pr}(0 \longleftrightarrow \infty)<\operatorname{Pr}(0 \longleftrightarrow \Pi) \leq \sum_{v \in \Pi} \operatorname{Pr}(A(v, \Pi))
$$

for each $\Pi$, implying

$$
p_{c} \geq p_{\text {cut }}^{\prime}:=\sup \left\{p: \inf _{\Pi} \sum_{v \in \Pi} \operatorname{Pr}(A(v, \Pi))=0\right\} .
$$

(Of course $p_{\text {cut }}^{\prime} \geq p_{\text {cut }}$.)

Question 3.1 Could it be that $p_{\text {cut }}^{\prime}(G)=p_{c}(G)$ for all $G$ ?

\section{Acknowledgement}

Thanks to Russ Lyons for a careful reading and several helpful comments.

\section{References}

[1] M. Aizenman and D.J. Barsky, Sharpness of the phase transition in percolation models, Comm. Math. Phys. 108 (1987), 489-526.

[2] G. Grimmett, Percolation (Second edition), Springer-Verlag, Berlin, 1999.

[3] R. Lyons, Random walks and percolation on trees, Ann. Probab. 18 (1990), 931-958.

[4] R. Lyons, The Ising model and percolation on trees and tree-like graphs, Comm. Math. Phys. 125 (1989), 337-353.

[5] R. Lyons and Y. Peres, Probability on Trees and Networks

[6] M.V. Menshikov, S.A. Molchanov and S.A. Sidorenko, Percolation theory and some applications, J. Soviet Math. 42 (1988), 1766-1810 (translated from Itogi Nauki $i$ Tekhniki 24 (1986), 53-110). 